\title{
Norois
}

Environnement, aménagement, société

\section{Influence de la suppression des petits barrages sur la végétation riveraine des cours d'eau du nord- ouest de la France}

Influence of small dam removal on riparian vegetation in northwestern France

\section{Doriane Depoilly et Simon Dufour}

\section{(2) OpenEdition}

Journals

Édition électronique

URL : https://journals.openedition.org/norois/5786

DOI : 10.4000/norois.5786

ISBN : 978-2-7535-5086-5

ISSN : $1760-8546$

\section{Éditeur}

Presses universitaires de Rennes

\section{Édition imprimée}

Date de publication : 31 décembre 2015

Pagination : 51-64

ISBN : 978-2-7535-5084-

ISSN : 0029-182X

\section{Référence électronique}

Doriane Depoilly et Simon Dufour, «Influence de la suppression des petits barrages sur la végétation riveraine des cours d'eau du nord-ouest de la France », Norois [En ligne], 237 | 2015, mis en ligne le 31 décembre 2017, consulté le 28 janvier 2022. URL : http://journals.openedition.org/norois/5786 ; DOI https://doi.org/10.4000/norois.5786 


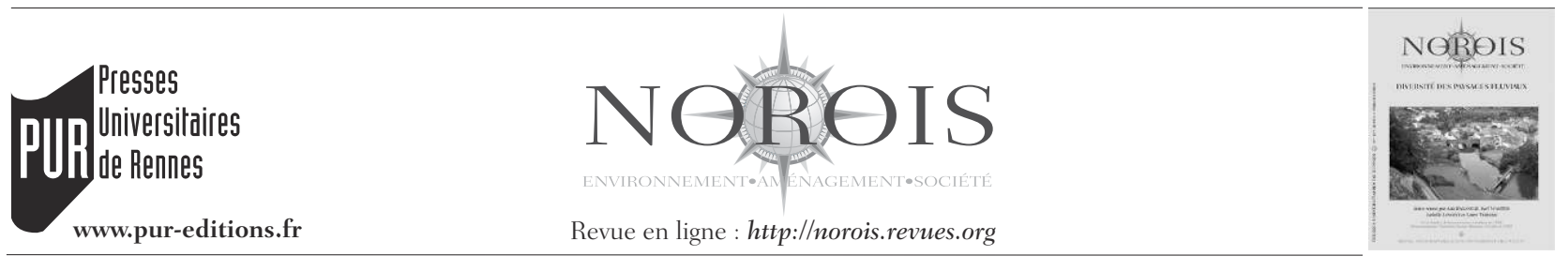

\title{
Influence de la suppression des petits barrages sur la végétation riveraine des cours d'eau du Nord-Ouest de la France
}

\author{
Influence of Small Dam Removal on Riparian Vegetation in Northwestern France
}

Doriane Depoilly ${ }^{\mathrm{a}}$, Simon Dufour ${ }^{\mathrm{a}^{*}}$

*Auteur correspondant : Tel : (33)299141846 (simon.dufour@univ-rennes2.fr)

a CNRS UMR LETG Rennes COSTEL, Université Rennes 2 - Place du Recteur-le-Moal, 35000 Rennes, France

Résumé : Au cours de la dernière décennie, la France s’est engagée dans une politique de restauration de la continuité écologique des cours d'eau. Cette politique se traduit notamment par la suppression de certains ouvrages (barrages, seuils de moulins, etc.) afin de favoriser le déplacement des espèces aquatiques et le transfert des sédiments. La multiplication des actions de suppression des obstacles à l'écoulement pose de nombreuses questions opérationnelles et scientifiques : acceptabilité, prise en compte des usages, influence réelle sur les hydrosystèmes, etc. Dans cette étude, nous analysons l'influence de ces actions sur un compartiment de l'hydrosystème souvent négligé, celui des milieux des plaines alluviales adjacentes au chenal. Pour cela, nous avons analysé l'influence d'une suppression de petits ouvrages sur la ripisylve située en amont pour deux cours d'eau de l'Ouest de la France (la Vire et l'Orne) par une approche de dendrochronologie. La mesure rétrospective des cernes de croissance des arbres de la ripisylve sur une période de trente ans met majoritairement en évidence une baisse significative de la croissance ligneuse suite à la suppression des ouvrages. Cette baisse est interprétée comme une réponse de la ripisylve à la modification locale de la position de la nappe phréatique suite à la suppression des ouvrages. De plus, la variabilité spatiale, la réponse différentielle des espèces et la temporalité de la réponse sont discutées. Ces résultats permettent de contribuer à la réflexion collective concernant les effets environnementaux de la politique de restauration de la continuité écologique des cours d'eau.

\begin{abstract}
Over the last decade, dam removal has been promoted to enhance biological continuity along French river systems. However, such policies raise many operational and scientific issues such as social acceptability, integration of various uses of rivers, the real impact on river ecosystems, etc. In this study, we analyze the influence of small dam removal on upstream floodplain habitats, which is usually a neglected component of such actions. For this purpose, we used dendrochronological analysis of riparian trees following two dam removals along the Vire and Orne rivers in northwestern France. Retrospective analysis of tree ring growth in a riparian forest over a thirty year period indicates a significant decrease in tree growth following dam removal. This decrease is interpreted as changes in hydrological connectivity following dam removal. We discuss the differing responses of species as well as the responses'spatial and temporal variabilities. The results contribute to the debate on environmental impacts of dam removal policies.
\end{abstract}

Mots clés : hydrosystème - restauration - ouvrages hydrauliques - dendrochronologie - ripisylve

Keywords: hydrosystem - restoration - hydraulic structures - dendrochronology - riparian vegetation 


\section{INTRODUCTION}

Au cours de la dernière décennie, la France s'est engagée dans une politique de restauration de la continuité écologique des cours d'eau. Cette politique s'inscrit dans la continuité de la directive cadre sur l'eau (DCE) de 2000, visant à améliorer l'état des masses d'eau des pays de l'union européenne, et des plans de gestion des espèces migratrices, comme par exemple le plan anguille (R[CE] n 1100/2007). Elle se traduit notamment par la suppression des obstacles à l'écoulement afin de favoriser le déplacement des espèces et le transfert des sédiments. Ces actions de suppression ont dans un certain nombre de cas, fait l'objet d'une opposition de la part des propriétaires d'ouvrages notamment sur la base d'arguments patrimoniaux, paysagers et liés aux usages de ces ouvrages (Jørgensen et Renöfält, 2012; Bellec et Lefebvre, 2012; Germaine et Barraud, 2013). L'existence même d'une controverse pose évidemment des questions sociales et politiques. Elle induit également un certain intérêt son influence réelle sur les hydrosystèmes. Or, l'évaluation de la réponse des milieux se concentre très majoritairement sur les milieux aquatiques, le chenal, (Zitek et al., 2008; Horreo et al., 2012; Chiu et al., 2013) et néglige le reste de la plaine alluviale (Shafroth et al., 2002). De fait, l'influence de la suppression des barrages sur la plaine alluviale reste en grande partie à évaluer, notamment dans le cas des petits ouvrages. Pourtant, les connaissances sur le fonctionnement hydroécologique de plaines alluviales permettent de supposer une influence non négligeable dans la mesure où la suppression des barrages se traduit par une modification significative de la position de la ligne d'eau (d'autant plus que le barrage est grand) (Pizzuto, 2002; Miller et Vizcaino, 2004). Or, la position de la nappe alluviale est étroitement liée à celle de la ligne d'eau (Piégay et al., 2003) et les écosystèmes alluviaux sont sensibles à la position de la ligne d'eau, comme cela a été démontré par l'analyse de la croissance et la régénération des espèces végétales riveraines (Amlin et Rood, 2003; Dufour et Piégay, 2008; Astrade et Dufour, 2010).

La suppression des ouvrages peut potentiellement entraîner différents impacts sur les milieux rivulaires. A l'aval, elle peut se traduire par une modification du régime hydrologique et un accroissement des apports en sédiments fins et en dias- pores (Shafroth et al., 2002). En amont de l'ouvrage, elle peut générer un abaissement du niveau de la nappe lié à la suppression de la retenue. En outre, la suppression du lac de barrage entraîne la mise à nu d'un espace anciennement inondé, sur lequel des peuplements pionniers et/ou des espèces invasives peuvent s'installer (Stanley et Doyle, 2003; Orr et Stanley, 2006). Géographiquement, les rares études menées sur l'impact de la suppression d'ouvrages sur la végétation riveraine ont été réalisées majoritairement aux Etats Unis et/ou sur des rivières à forte énergie (Orr et Stanley, 2006; Auble, 2007) dans des contextes de faible occupation humaine des plaines alluviales. Elles sont encore peu nombreuses dans des régions tempérées comme l'ouest de la France, où les formes fluviales, les régimes hydrologiques et le contexte humain diffèrent fortement des cours d'eau étudiés jusqu'à présent.

L'objectif de cette étude est de mettre en évidence l'influence éventuelle d'une suppression d'ouvrages sur la ripisylve des cours d'eau du nord-ouest de la France grâce par une approche de dendrochronologie. Plus spécifiquement, il s'agit d'explorer la réponse de la croissance des arbres de la ripisylve en fonction des espèces et de la localisation des individus par rapport à l'ouvrage. Notre hypothèse de travail est que la suppression d'un ouvrage, en entrainant une disparition de la retenue d'eau et une baisse du niveau de la ligne d'eau en amont, doit avoir un impact sur les conditions de croissance des arbres (Willms et al., 1998). L'effet attendu est une baisse de la croissance notamment pour les espèces de la ripisylves exigeantes en eau (ex. Alnus glutonisa). La dendrochronologie est classiquement utilisée afin de déterminer la chronologie, l'intensité et la répartition spatiale des changements passés (Braam et al., 1987). Cependant, la sensibilité de cet outil est variable dans le temps et dans l'espace (Hupp et al., 2016). Cette étude comprend donc une dimension méthodologique, puisqu'il s'agit de tester l'intérêt effectif d'une approche dendrochronologique pour étudier le fonctionnement des ripisylves des cours d'eau du Nord-Ouest de la France ce qui, à notre connaissance, n’a jamais été fait. 


\section{Matériel et MÉTHOdes}

\section{Sites d'étude}

L'étude a été réalisée sur 2 sites localisés dans les bassins versant de l'Orne et de la Vire : respectivement le site du moulin du Viard (Calvados) et du moulin de Rondelles (Manche) (figure 1). L'Orne et la Vire sont des fleuves côtiers bas-normands dont les principales caractéristiques sont présentées dans le tableau 1. Au sein du réseau hydrographique de chaque cours d'eau, le site d'étude a été sélectionné sur la base de trois critères : une suppression la plus ancienne possible, la présence d'une ripisylve en amont de l'ancien barrage et la présence d'une végétation aussi spontanée que possible. Le premier critère permet de disposer d'un temps de réponse potentielle suffisant car l'effet de la modification des conditions environnementales sur la croissance ligneuse est parfois décalé dans le temps de quelques années (Tardif et Bergeron, 1997 ; Dufour, 2005). Dans les deux cas étudiés, les ouvrages ont été supprimés en 1997; il s’agit de seuils de moulins d'une hauteur respectivement de 2 et $3 \mathrm{~m}$ pour le site du moulin du Viard et celui du moulin de Rondelles. Le troisième critère, c'est-àdire la présence d'une végétation aussi spontanée que possible, a été appréciée à dire d'expert sur le terrain. Ainsi, dans la mesure du possible, les individus sélectionnés n'ont pas trop subie de tailles; pour cela ont été exclus les arbres avec des traces de coupe ou d'élagage récent et les individus taillés en têtards.

La dynamique de l'occupation des deux sites a été retracée par photo-interprétation des clichés IGN depuis 1955 (Depoilly, 2014). Elle est caractérisée par une tendance au recul des milieux ouverts, essentiellement des praires, et à l'accroissement des superficies boisées qui passe de $8 \%$ à $24 \%$ (moulin des Rondelles) et de $41 \%$ à $55 \%$ (moulin du Viard). Plus de $85 \%$ de cette progression est observée avant la suppression des ouvrages, c'est-à-dire sur la période 1955-1997.
Figure 1 : Localisation A) des bassins au sein du bassin Seine Normandie et B) des sites d'études au sein des bassins de l'Orne et de la Vire (fond MNT IGN)

Location of

A) the Orne and Vire watersheds within the SeineNormandy basin and B) study sites (DEM source: IGN)

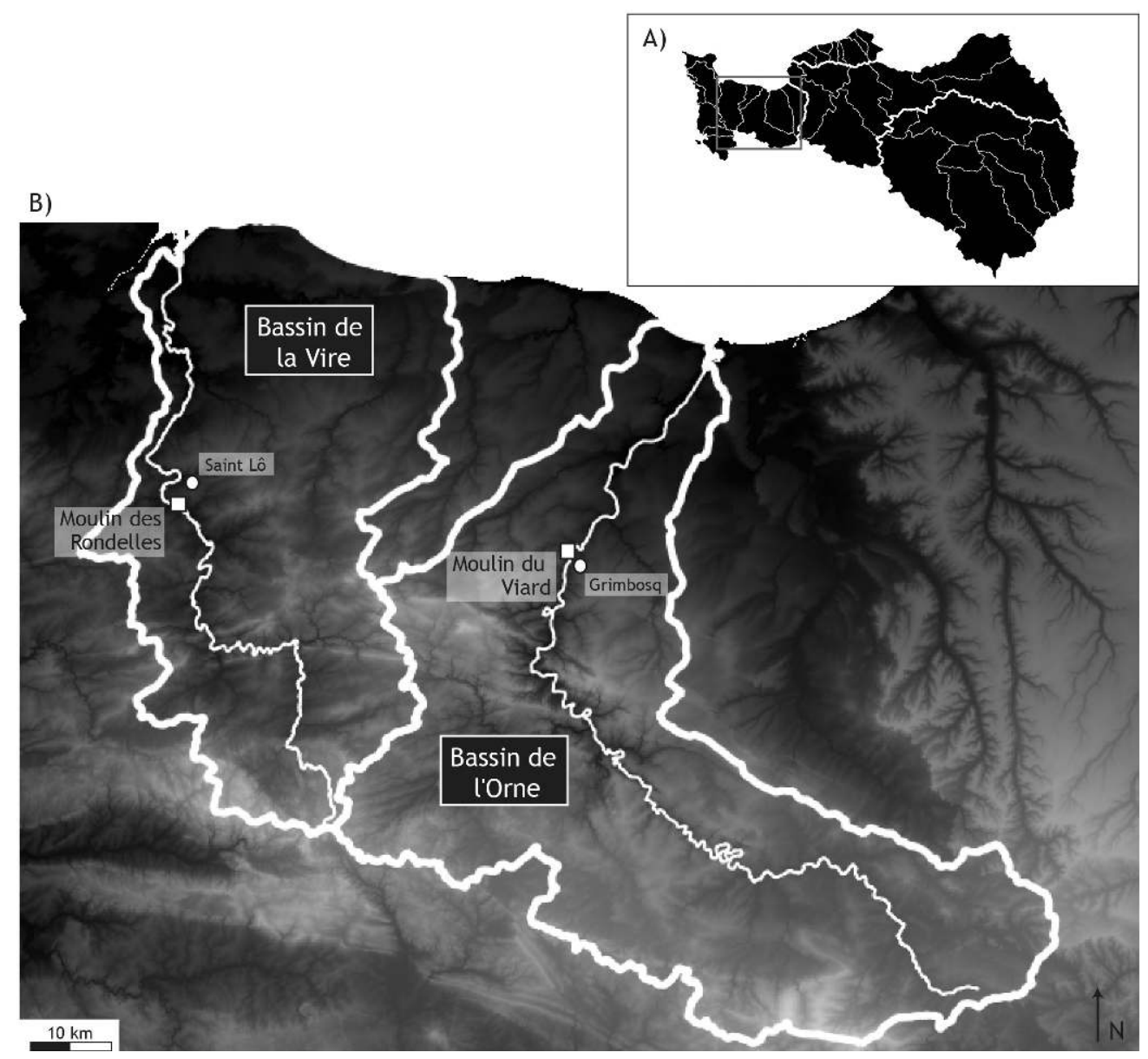




\begin{tabular}{|c|c|c|}
\hline Cours d'eau & Orne & Vire \\
\hline Longueur $(\mathrm{km})$ & 170 & 128 \\
\hline Superficie du bassin $\left(\mathrm{km}^{2}\right)$ & 2930 & 1970 \\
\hline Contexte géologique & $\begin{array}{l}\text { Briovérien et Cambiren (amont), Juras- } \\
\text { sique (aval) }\end{array}$ & $\begin{array}{l}\text { Briovérien et Cambrien (amont), dépôts } \\
\text { quaternaires (aval) }\end{array}$ \\
\hline Module $\left(\mathrm{m}^{3} \cdot \mathrm{s}^{-1}\right)^{*}$ & 24,7 & 12,7 \\
\hline Module spécifique $\left(1 . \mathrm{s}^{-1} \cdot \mathrm{km}^{2}\right)^{*}$ & 9,9 & 14,8 \\
\hline$Q_{10}\left(\mathrm{~m}^{3} \cdot \mathrm{s}^{-1}\right)^{*}$ & 320 & 180 \\
\hline Site & Moulin du Viard & Moulin des Rondelles \\
\hline Localisation, par rapport à la source $(\mathrm{km})$ & 140 & 80 \\
\hline Contexte écologique & ZNIEFF types 1 et 2 & $\begin{array}{l}\text { ZNIEFF type } 2 \text {, Arrêté de protection de } \\
\text { biotope }\end{array}$ \\
\hline Pente du tronçon $\left(\mathrm{m} \cdot \mathrm{km}^{-1}\right)$ & $\cong 2$ & $\cong 1$ \\
\hline Largeur du chenal (m) & $25-35$ & $15-25$ \\
\hline Hauteur du barrage $(\mathrm{m})$ & 2 & 3 \\
\hline Date de suppression & 1997 & 1997 \\
\hline
\end{tabular}

* données hydrologiques pour les stations du moulin des Rondelles (1971-2014) et de May sur Orne (1983-2014)

Tableau 1 : Présentation générale des cours d'eau et des sites étudiés General characteristics of studied streams and sites

\section{Principe général de l'approche dendrochronologique}

Afin d'identifier une influence éventuelle de la suppression des ouvrages sur la végétation rivulaire, une approche dendrochronologique a été mise en œuvre. Celle-ci est basée sur le principe que les cernes de croissance produits par les espèces ligneuses enregistrent une variabilité interannuelle de leur largeur et que cette variabilité est liée à la dynamique des paramètres environnementaux influençant la croissance ligneuse (climat, apport en eau, ouverture ou fermeture du milieu...) (Béguin et al., 1991; Stoffel et al., 2012). Dans le cas des forêts riveraines, les facteurs environnementaux dominants sont, premièrement, le degré de connexion hydrologique avec le chenal et la nappe d'accompagnement et, secondairement, les processus sédimentaires qui ont cours dans le chenal et dans la plaine (Astrade et Bégin, 2003; Astrade et Dufour, 2010; Hupp et al., 2016). De fait, la végétation alluviale est un bon indicateur de la dynamique des cours d'eau. L'approche dendrochronologique a ainsi été mise en œuvre par de nombreux auteurs afin d'identifier la dynamique des métamorphoses fluviales (Bravard et al., 1989), le changement du régime des crues et des conditions d'alimentation en eau (Astrade et
Béguin, 1997; Smelko et Scheer, 2000), la mobilité du chenal principal (Amlin et Rood, 2003; Dufour et Piégay, 2008; Stella et al., 2013) ou la dynamique de la nappe phréatique (Dufour, 2005).

Afin de différencier les impacts liés à la suppression de barrages des autres facteurs extérieurs, les variables externes sans rapport avec la suppression mais pouvant influencer la croissance des arbres ont été prises en compte. De fait, les chroniques des conditions climatiques et hydrologiques enregistrées sur nos deux sites ont été analysées sur une période de 34 ans (1980 à 2013) afin d'identifier d'éventuelles ruptures dans ces chroniques sur l'ensemble de la période d'étude. Concrètement, les variables suivantes ont été prises en compte : la moyenne des précipitations mensuelles, ainsi que des températures minimales et maximales mensuelles enregistrées aux stations météorologiques de Météofrance (Freney-le-Vieux et Condé-sur-Vire) proches des deux sites d'études. À partir de ces données, nous avons réalisé, à l'instar de Dufour (2005) et de Singer et al. (2012), la moyenne des températures minimales et maximales ainsi que le cumul des précipitations pour chaque année et pour la saison d'activité végétale de chaque année (période mars/septembre). En parallèle, à partir des débits 
mensuels de chaque cours d'eau estimés au niveau des stations hydrologiques des 2 sites (moulin des Rondelles et May sur Orne; banque hydro), une moyenne annuelle des débits et une moyenne pendant la saison d'activité végétale ont été effectuées.

\section{Stratégie d'échantillonnage et traitement des carottes dendrochronologiques}

Sur chaque placette, des prélèvements dendrochronologiques ont été réalisés au sein des quatre placettes (figure 2) : trois placettes positionnées le long d'un axe amont-aval (V3 à V1 et R3 à R1) et une placette témoin (V0 et R0), peu ou pas impactée par la suppression de l'obstacle.
Les placettes d'échantillonnage sont localisées respectivement à 20,350 et 538 mètres pour le moulin des Rondelles et à 114, 412 et 560 mètres en amont de l'ancien barrage pour le site du moulin du Viard. À notre connaissance, il n'existe pas de données topographiques utilisables pour la période avant la suppression, la baisse de la ligne d'eau au droit des placettes ne peut donc être estimée que sur la base de la hauteur des ouvrages et de la pente du tronçon (Tableau 1). Cette estimation donne des valeurs de 2,98, 2,65 et 2,46 mètres respectivement pour les placettes (R1, R2 et R3) du site du moulin des Rondelles et de 1,77, 1,18 et 0,88 pour les placettes $(\mathrm{V} 1, \mathrm{~V} 2$ et $\mathrm{V} 3)$ du site du moulin du Viard.
Figure 2 : Localisation des placettes dendrologiques au sein des sites d'étude, A) Moulin des Rondelles, B) Moulin du Viard (source : BD ORTHO, IGN)

Dendrochronological plot location within the sites of A) Moulin des Rondelles and B) Moulin du Viard (image source: IGN)

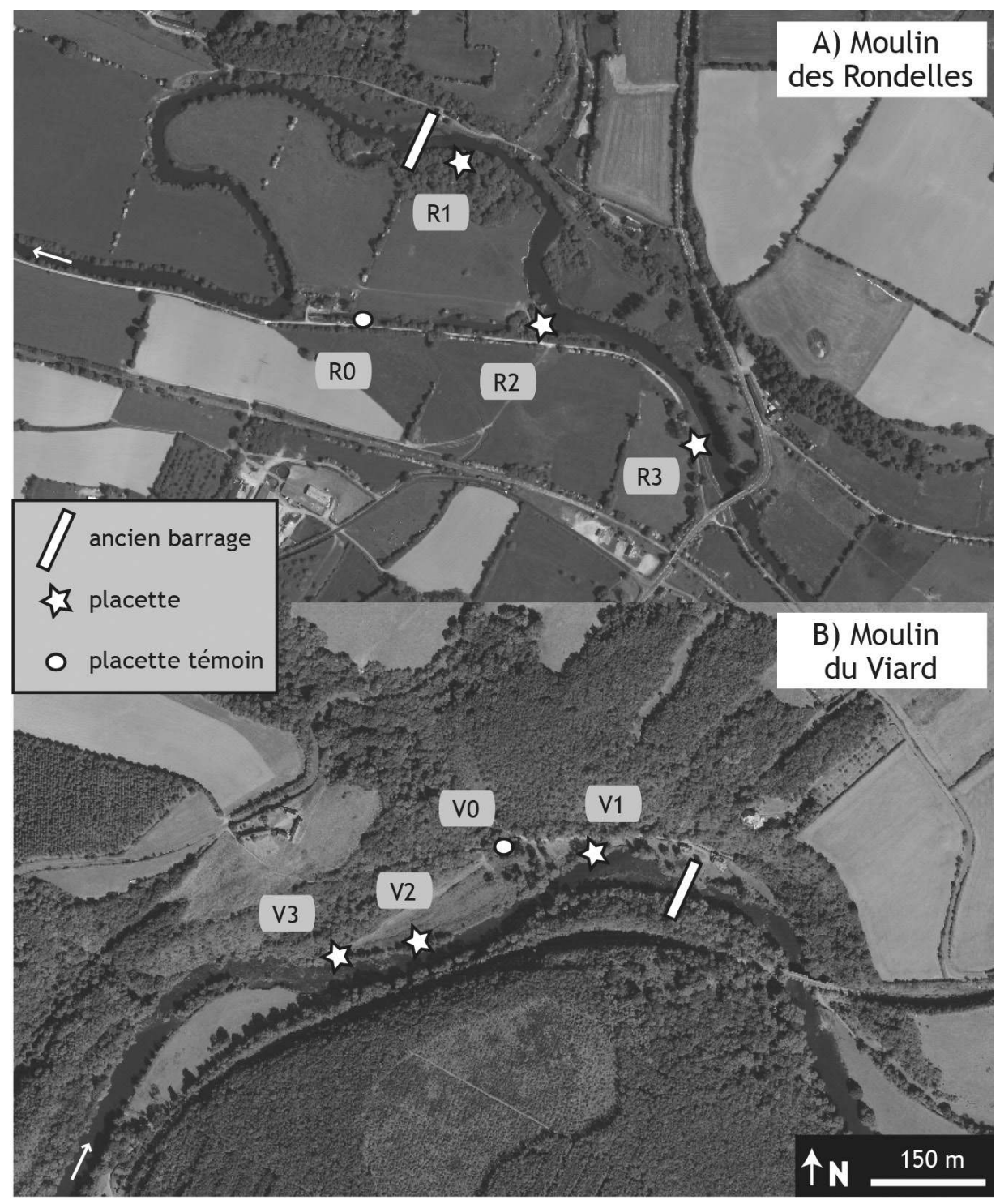


Les placettes témoins ont été sélectionnées sur la base de trois critères : être composée d'individus de l'espèce la plus fréquente sur les berges des deux sites (i.e. l'aulne), être située à proximité des autres placettes afin de connaitre les mêmes conditions microclimatiques et avoir été le moins possible influencée par la suppression de l'ouvrage. Ce dernier point, le plus délicat compte tenu de l'ancienneté des travaux et de l'absence d'observations réalisées avant les travaux, a été évalué à dire d'expert in situ. Pour le site du moulin des Rondelles, la placette témoin a été positionnée sur un canal dont le niveau d'eau est contrôlé par un ouvrage d'art (une écluse) encore en place. Pour le site du moulin de Viard, la placette témoin a été positionnée le long d'un petit affluent de l'Orne; sa position altimétrique (i.e. environ 5 mètres au-dessus du niveau de la berge de l'Orne permet légitimement d'exclure une éventuelle influence de la suppression.

Pour chaque site, 18 arbres de différentes espèces ont été échantillonnés, avec un minimum de 3 par placette (Mäkinen et Vanninen, 1999). Les individus dominants dans la ripisylve ont été préférentiellement sélectionnés. Les espèces échantillonnées sont des aulnes glutineux (Alnus glutinosa, $\mathrm{n}=21$ ), érables sycomores (Acer pseudoplatanus, $\mathrm{n}=3$ ), frênes communs (Fraxinus excelsior, $\mathrm{n}=7$ ) et des tilleuls à grandes feuilles (Tilia platyphyllos, $\mathrm{n}=5$ ). Chaque individu a fait l'objet de 2 prélèvements à 1,30 m du sol à l'aide d'une tarière de Pressler (un en direction de l'amont et un de l'aval). Chaque échantillon a été découpé et poli afin de permettre une meilleure lecture des cernes (Rubino et al., 2004). Les mesures des cernes ont été réalisées en laboratoire via le logiciel TSAP-win (CREAAH Université Rennes 1).

Lors des prélèvements, des relevés topographiques ont été réalisés afin de déterminer position altimétrique des arbres par rapport au chenal (Munaut, 1979).

\section{L'analyse des données}

Les chroniques climatiques, hydrologiques et dendrologiques ont été analysées par des tests statistiques de Pettitt et de Hubert afin d'évaluer l'existence de ruptures significatives. Pour ces tests d'homogénéité, l'hypothèse nulle est l'homogénéité de la série temporelle. Schématiquement, ils per- mettent donc de segmenter une série en plusieurs périodes de telle sorte que la moyenne calculée sur toute période soit significativement différente de la moyenne de la période voisine (Lubes et al., 1994). Les arbres considérés comme trop jeunes par rapport à la date des suppressions ont été écartés (moins de 20 ans) (Munaut, 1979). De plus, la comparaison de la croissance des cernes après et avant la suppression a été évaluée par un test non paramétrique de significativité (test de Kruskal Wallis).

\section{RÉsultats}

\section{Dynamique des facteurs hydroclimatiques}

L'analyse des chroniques hydrologiques et climatiques ne met pas évidence de rupture significative et durable au cours de la période d'étude (19802013) sauf pour la température maximale qui augmente à partir de 1989 (tableau 2). Les deux tests indiquent des résultats légèrement différents avec plus de ruptures significatives identifiées par le test de Hubert. Ces résultats sont cohérents avec les observations de Dubreuil et al. (2012).

\section{Dynamique individuelle de la croissance ligneuse}

Premièrement, la comparaison de la croissance des cernes avant et après la suppression des barrages met en évidence trois situations différentes : l'augmentation statistiquement significative, la stagnation ou la diminution statistiquement significative de la croissance radiale. De fait, 30 échantillons sur 36 enregistrent une différence significative statistiquement sur nos deux sites. La suppression a ainsi entraîné un changement de croissance (positif ou négatif) pour près de $83 \%$ des individus avec $66 \%$ de diminution, $17 \%$ de stagnation et $17 \%$ d'augmentation. Sur les 18 arbres échantillonnés au moulin des Rondelles, 2 enregistrent une augmentation, 13 voient leur croissance diminuer de manière significative et 3 arbres ne semblent pas être impactés par la suppression du barrage. Pour le site du moulin du Viard, la diminution de la croissance concerne 11 arbres, l'augmentation 4 arbres et la stagnation 3 arbres. 


\begin{tabular}{|l|c|c|c|c|}
\hline & \multicolumn{2}{|c|}{ Test de Pettitt } & \multicolumn{2}{c|}{ Test de Hubert } \\
\hline Variable & Site Rondelles & Site Viard & Site Rondelles & Site Viard \\
\hline Débit moyen annuel & NS & NS & NS & NS \\
\hline $\begin{array}{l}\text { Débit moyen période d'activité } \\
\text { végétale }\end{array}$ & NS & NS & NS & NS \\
\hline Précipitations totales & NS & NS & NS & $\begin{array}{c}\text { Augmentation } \\
\text { après 1989 et baisse } \\
\text { après 1992 }\end{array}$ \\
\hline $\begin{array}{l}\text { Précipitations période d'activité } \\
\text { végétale }\end{array}$ & NS & NS & NS & NS \\
\hline $\begin{array}{l}\text { Température minimale (moyenne } \\
\text { annuelle) }\end{array}$ & NS & NS & $\begin{array}{c}\text { Augmentation } \\
\text { après 1999 et baisse } \\
\text { après 2001 }\end{array}$ & NS \\
\hline $\begin{array}{l}\text { Température minimale (moyenne } \\
\text { période d'activité végétale) }\end{array}$ & NS & $\begin{array}{c}\text { Augmentation après } \\
1989\end{array}$ & $\begin{array}{c}\text { Augmentation } \\
\text { après 1989 } \\
\text { après 1989 } 1989\end{array}$ & $\begin{array}{c}\text { Augmentation } \\
\text { après 1989 }\end{array}$ \\
\hline $\begin{array}{l}\text { Température maximale (moyenne } \\
\text { annuelle) }\end{array}$ & $\begin{array}{c}\text { Augmentation } \\
\text { après 1989 }\end{array}$ \\
\hline $\begin{array}{l}\text { Température maximale (moyenne } \\
\text { période d'activité végétale) }\end{array}$ & & NS \\
\hline
\end{tabular}

Tableau 2 : Tests de rupture appliqués aux chroniques des variables hydrologiques et climatiques (seuil de significativité à $5 \%$; NS = pas de ruptures significatives) Statistical threshold tests of hydrologic and climatic series ( $p<5 \%$; NS = no significant thresholds)

Deuxièmement, les tests de rupture appliqués aux chroniques de la croissance des cernes permettent d'identifier respectivement 25 (test de Pettitt) et 30 individus (test de Hubert) sur 36 pour lesquels la croissance change significativement au cours de la période d'étude. La majorité de ces ruptures sont des ruptures à la baisse : respectivement 77 et $80 \%$ selon le test. Temporellement, les ruptures statistiquement significatives après 1997 sont majoritaires sur les deux sites (figure 3) puisque les tests de Pettitt et Hubert recensent chacun 21 arbres dans cette situation, soit $60 \%$ des arbres ou $70 \%$ si l'on exclut les arbres des placettes témoins. Pour le site du moulin des Rondelles, selon le test la période enregistrant le plus de baisses est 1997/2008 (test de Pettitt) ou 1997/2000 (test de Hubert). Pour le site du moulin du Viard, dans les deux cas, il s'agit de la période 2001/2004.

\section{Effet de la localisation et différences interspécifiques}

Compte tenu de la distribution spatiale des espèces, il est difficile d'analyser séparément les effets de localisation (distance à l'ouvrage, position sur la berge) et la réponse spécifique. En effet, il n’a pas été possible de trouver les mêmes espèces sur chaque site, les deux variables ne peuvent pas donc être considérées comme indépendantes.

Concernant la croissance des arbres des placettes témoins, les chroniques des valeurs moyennes (figure 4) mettent en évidence l'absence de changements significatifs pour le site du moulin du Viard et une rupture à la baisse pour le site du moulin des rondelles, mais datée de 1989.

En ce qui concerne la distance à l'ouvrage, sur le site du moulin des Rondelles, toutes les placettes voient la croissance moyenne des arbres diminuer après la suppression du barrage quelques soit l'espèce (figure 4). La date de la rupture significative (1998) est la même pour les aulnes de la placette la plus proche $(\mathrm{R} 1)$ et pour les frênes de la placette la plus éloignée (R3). Sur la placette R1, la rupture pour les érables intervient après celle des aulnes, respectivement 2003 (ou 2005 selon le test) et 1998. Les aulnes de la placette intermédiaire (R2) enregistrent une baisse significative juste après ceux de la placette Rl (1999). Sur le site du moulin du Viard, les ruptures significatives se produisent à peu près au moment de la suppression du barrage (figure 4), mais les aulnes des placettes V2 et V3 enregistrent des baisses, alors que les tilleuls et les frênes de la placette 1 enregistrent une augmentation de leur croissance radiale. 


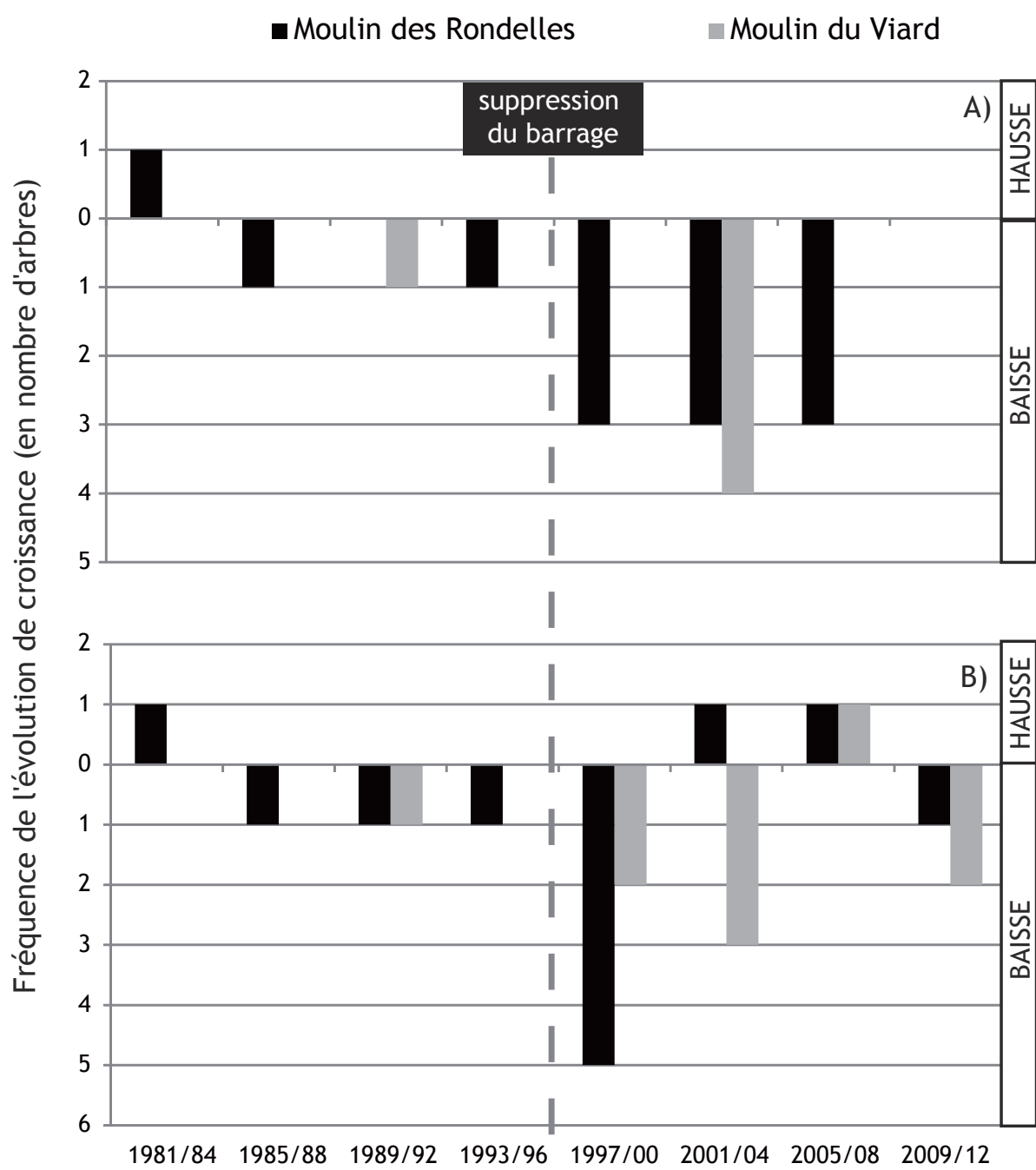

Figure 3 : Période d'occurrence des ruptures significatives de la croissance des arbres des 2 sites selon les tests A) de Pettitt et B) de Hubert. Les valeurs indiquent le nombre d'individus enregistrant respectivement une augmentation ou une diminution de la croissance Periods during which significant thresholds in tree growth were passed, based on: A) Pettitt test and B) Hubert test. Values indicate the number of individual trees exhibiting an increase or decrease in growth

En ce qui concerne la position des arbres sur la berge, la comparaison des altitudes relatives des individus et des différences de croissance après/ avant la suppression est également délicate à analyser. En effet, les aulnes sont presque toujours situés plus bas sur les berges que les autres espèces (figure 5). De plus, il n'existe pas de relation statistique simple et significative entre ces variables : des individus de la même espèce peuvent enregistrer des différences de croissance très dissemblables malgré une position altitudinale proche. Par exemple, les quatre aulnes situés à $1,5 \mathrm{~m}$ sur le site du moulin des Rondelles appartiennent tous à la placette 2, ils ont pourtant des comportements très variables puisque le différentiel de croissance fluctue entre 200 et $-350 \mathrm{~mm}$.

\section{Discussion}

Si les résultats obtenus dans le cadre de cette analyse de la réponse de la végétation riveraine à la suppression de petits barrages dans le Nord-Ouest de la France par dendrochronologie ne répondent que partiellement à la question scientifique qui l'a suscitée, ils permettent néanmoins d'apporter des éléments de discussion sur trois aspects : le fonctionnement hydroécologique des plaines des alluviales des cours d'eau du Nord-Ouest de la France, la pertinence de l'approche dendrochronologique dans l'évaluation de ce fonctionnement et la complexité des enjeux politiques et opérationnels dans les opérations de suppression d'ouvrages hydrauliques en rivière. 


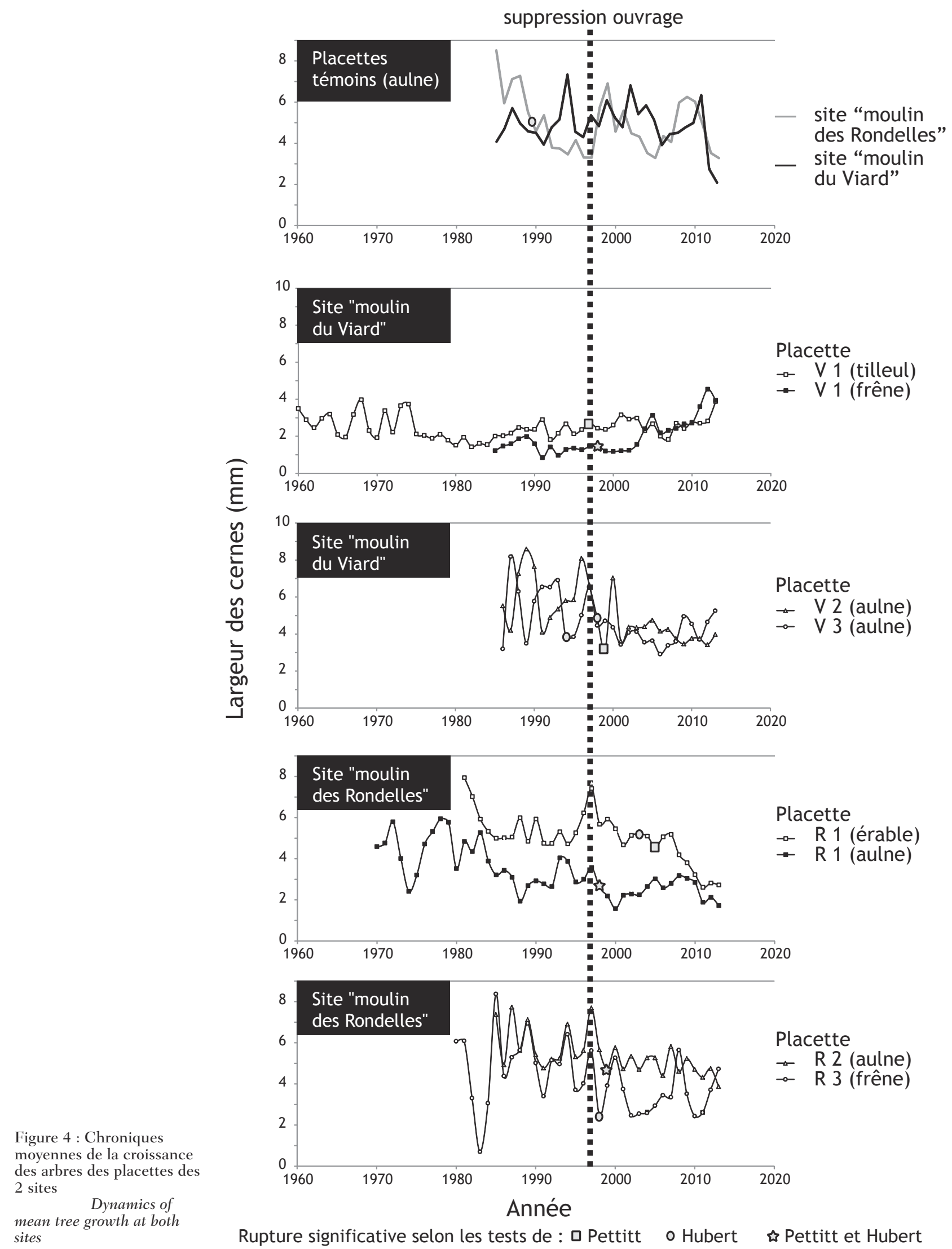




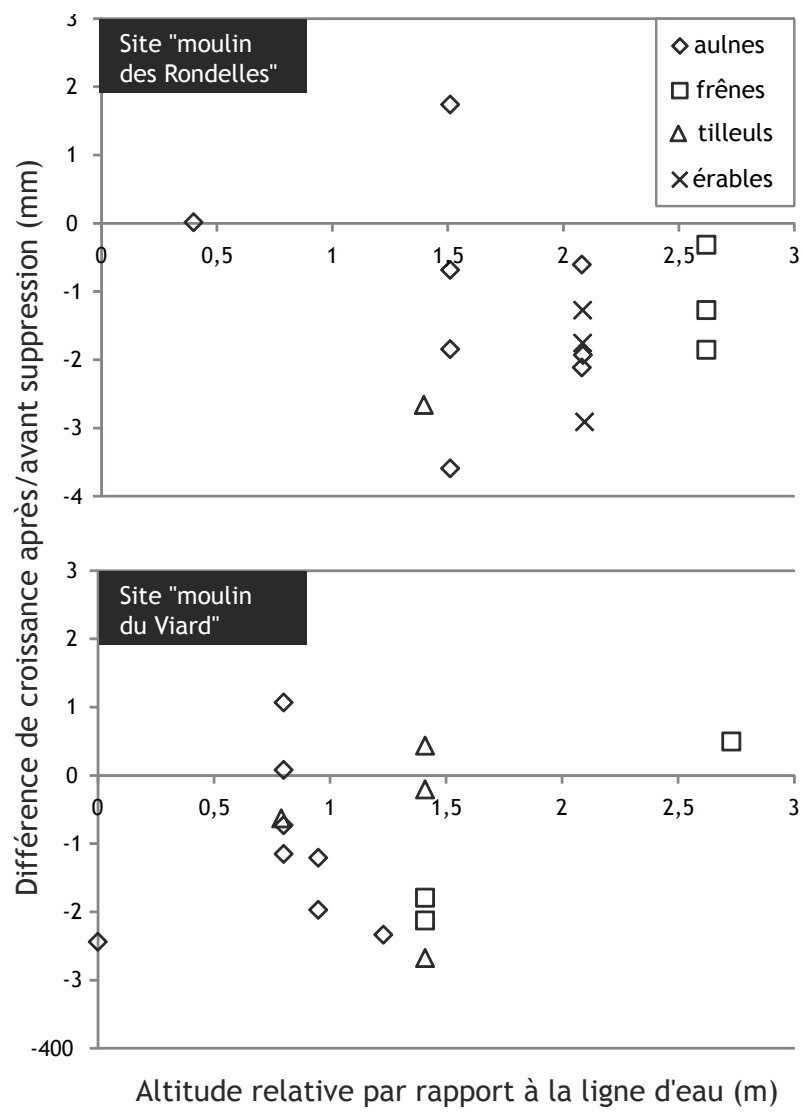

Figure 5 : Relation entre l'altitude relative des arbres par rapport à la ligne d'eau et la différence de croissance radiale entre la période après la suppression du barrage et avant la suppression (les arbres des placettes témoins ne sont représentés)

Relation between relative elevation (above the main channel) and difference in radial growth before dam removal and after dam removal (trees from control plots are not included)

\section{Fonctionnement hydroécologique des plaines des alluviales}

En ce qui concerne le fonctionnement hydroécologique des plaines alluviales des cours d'eau du Nord-Ouest de la France, nos résultats semblent démontrer une réponse sensible des arbres constitutifs de la ripisylve à la suppression de barrages d'environ deux à trois mètres de haut. Cette réponse est enregistrée dans le tronçon situé en amont de l'ancien ouvrage. Le rôle du barrage peut être mis en avant dans la mesure où la majorité des arbres enregistrent des différences significatives de croissance radiale avant et après la suppression et que ni les autres facteurs hydroclimatiques (débit, précipitations) ni les placettes témoins ne montrent de changements temporellement concordant avec les ruptures de cette croissance. Ainsi, parmi les facteurs hydroclimatiques, seules les températures connaissent un changement à la hausse et celuici intervient presque 10 ans avant les changements observés dans la croissance des arbres. L'existence de ce décalage temporel et la prédominance des facteurs locaux dans le contrôle de la croissance des arbres de plaine alluviale (Dufour, 2005) permettent de penser légitimement que ce facteur ne joue pas un rôle prépondérant.

Le changement majoritaire dans la croissance des arbres est une baisse (66\%) de celle-ci, mais certains individus enregistrent une augmentation $(17 \%)$. Les deux sites réagissent légèrement différemment puisque plus d'individus enregistrent un changement significatif sur le site du moulin des Rondelles $(73 \%$ de diminution et $11 \%$ d'augmentation) que sur celui du moulin du Viard $(61 \%$ de diminution et $23 \%$ d'augmentation). En l'état des observations, la différence la plus marquante entre les deux sites semble être la dimension des ouvrages puisque celui du moulin des Rondelles mesurait $3 \mathrm{~m}$ contre $2 \mathrm{~m}$ pour celui du moulin du Viard. Il convient cependant de noter que les changements de croissance, bien que significatifs, restent modérés en amplitude (figure 4) comparés, par exemple, aux observations de Shafroth et al. (2000) le long la Bill Williams River dans l'Arizona qui observent de très fortes mortalités pour des mouvements de nappe à partir d'un mètre de baisse ou de Dufour (2005) sur des frênes de la plaine du Rhône au niveau de l'île de la Platière dont la croissance a été réduite de moitié suite à des baisses d'environ un à deux mètres du niveau de la nappe alluviale. Aucun élément tangible ne permet d'expliquer cette différence, mais nous pouvons formuler plusieurs hypothèses. Premièrement, la diminution du niveau de la nappe phréatique a pu être atténuée par une épaisseur importante de sédiments fins dans les berges. En effet, les sondages effectués à la tarière pédologique attestent de dépôts essentiellement limono-argileux sur des épaisseurs toujours supérieures à 2 mètres. Or ce type de substrat maintient une quantité d'eau disponible importante (que l'eau provienne de la nappe ou des précipitations) et limiterait l'effet de la diminution du niveau de la ligne d'eau par un effet tampon. Deuxièmement, la région d'étude connaît des conditions climatiques moins contrastées et moins contraignantes pour les 
espèces végétales que les contextes méditerranéens, semi-arides et/ou de tête de bassin versant dans lesquels la plupart de ces études ont été réalisées. De fait, l'impact sur la dynamique de la ripisylve à moyen terme est difficile à inférer sur la base de ces seuls résultats.

La position spatiale longitudinale, l'altitude relative par rapport à la ligne d'eau et l'espèce d'appartenance des individus sont des facteurs de contrôle dont l'influence est délicate à analyser. En effet, pour des raisons méthodologiques, il n’a pas été possible d'établir une stratégie d'échantillonnage permettant de tester chaque facteur indépendamment des autres. En l'état, les résultats indiquent l'absence de lien entre la position altimétrique par rapport au chenal et la modification de croissance des arbres, contrairement par exemple aux observations de Dufour (2005) sur le frêne. Mais la distribution des espèces génère un biais dans la mesure où les aulnes sont majoritairement situés plus bas que les autres espèces (ce qui est cohérent avec les caractéristiques écologiques de cette espèce).

En ce qui concerne l'influence de la position des placettes par rapport à l'ancien ouvrage, elle n'a pas été mise en évidence. En effet, les tests de rupture appliqués aux chroniques des croissances moyennes mettent en évidence une relative synchronicité des dates de rupture pour l'ensemble des placettes. Ce résultat pourrait s'expliquer par l'absence de prise en compte dans la stratégie d'échantillonnage de placette localisée très loin de l'ancien ouvrage puisque le maximum est ici de 550 mètres. Contenu de la pente des tronçons (tableau 1), cela génère des différences en matière d'abaissement de la ligne d'eau relativement faibles entre les placettes (au maximum $0,5 \mathrm{~m}$ pour le moulin des Rondelles et $0,9 \mathrm{~m}$ pour le moulin du Viard).

Enfin, concernant les différences interspécifiques, les espèces de bois dur (érable, frêne, tilleul) se distinguent parfois de l'aulne. La différence peut intervenir par une réponse plus tardive, comme par exemple avec les érables de la placette $1 \mathrm{du}$ moulin des Rondelles, ou par une réponse inverse, comme par exemple les tilleuls et les frênes du site du moulin du Viard pour lesquels la croissance est plus forte après suppression du barrage et non plus faible comme pour les aulnes. Ces différences peuvent s'expliquer par des exigences écologiques, des fonctionnements physiologiques et des capacités de mobilisations de la ressources en eau différents (Besnard, 1987; Dufour, 2005 ; Singer et al., 2012). Un approfondissement de cette question par l'utilisation des isotopes de l'eau semble une perspective intéressante pour analyser plus en détail ces différences. De même, l'absence de suivi piézométrique de l'impact de la suppression des barrages est frein à la bonne compréhension des résultats. La réalisation de suivis avant/après d'un nombre significatif d'opérations permettrait de combler cette lacune.

\section{Approche dendrochronologique du fonctionnement hydroécologique des plaines alluviales}

Sur le plan méthodologique, notre étude confirme la pertinence de l'approche dendrochronologique dans l'évaluation de la réponse fonctionnelle des habitats de plaine alluviale à la dynamique du chenal et des nappes associées (Bravard et al., 1989; Astrade, 1996, Amlin et Rood, 2003; Astrade et Dufour, 2010; Stella et al., 2013). Cependant, certains éléments rendent son application dans le contexte des petits cours d'eau à énergie faible ou modérée du nord-ouest de la France probablement plus délicate que dans les contextes où elle a été essentiellement utilisée jusqu'à présent : climats secs et/ou rivières à forte énergie (Stromberg et Patten, 1996; Shafroth et al., 2000 ; Disalvo et Hart, 2002; Galuszka et Kolb, 2002; Stella et al., 2013) ou plaines alluviales de rivières à faible énergie avec de longues périodes d'inondation, notamment en climat sub-tropical, durant lesquelles l'excès d'eau contrôle la croissance (Reilly et Johnson, 1982; Mitsch et Rust, 1984; Golet et Lowry, 1987; Keeland et al., 1997). Premièrement, l'espèce la plus fréquente sur le long des cours étudiés est l'aulne glutineux; or celle-ci est connue pour une présence non négligeable de faux cernes qui rend la lecture des échantillons délicate (Moulin, 2005). Deuxièmement, la faible densité des arbres au sein des ripisylves de ces cours d'eau empêche, dans de nombreux cas, d'atteindre un nombre satisfaisant d'individus pour un site ayant des conditions hydromorphologiques homogènes, et donc de disposer d'un échantillon robuste en ce qui concerne le nombre d'individus. Enfin, troisièmement, les résultats des deux tests statistiques employés afin d'identifier les dates des ruptures statistiquement significatives sont sen- 
siblement différents. Ainsi, la date de rupture est globalement plus tardive selon le test de Pettitt par rapport au test de Hubert. Un travail fin d'évaluation de la qualité de ces tests reste donc à produire, travail malheureusement impossible à réaliser avec notre jeu de données.

\section{Complexité des opérations de suppression d'ouvrages hydrauliques en rivière}

Enfin, les résultats de cette étude illustrent en partie la complexité des enjeux politiques et opérationnels qui s'articulent autour de la stratégie de restauration de la continuité écologiques des cours d'eau par suppression des ouvrages de type seuils ou petits barrages. En effet, cette stratégie soulève la question de notre capacité à combiner les effets de telles opérations sur des plans multiples, relevant des dimensions écologistes et socio-culturelles. La mobilisation de critères multiples, si elle assure une intégration plus complète des différents enjeux, ne résout pas les éventuelles oppositions. Ainsi, en ce qui concerne la dimension écologique, la restauration de la continuité se concentre sur le chenal, dont elle vise à améliorer les conditions biotopiques et, dans de nombreux cas, elle n'intègre pas les écosystèmes de plaine alluviale, dont la réponse elle-même peut être variable. Par exemple, pour les espèces de la ripisylve, la suppression d'un barrage peut se traduire par une recolonisation d'espèces, avec accroissement de la biomasse et de la richesse spécifique (Isaksson, 2010; Kim et al., 2014). Dans le même temps, nos résultats semblent montrer que le risque d'une perte partielle du caractère humide des habitats riverains en place existe, même si l'ampleur du phénomène reste à analyser plus finement. Si le bilan écologique est difficile à établir pour une seule composante du système, il devient vite évident que le bilan global, intégrant plusieurs systèmes (écosystemes et sociosystèmes), à différentes échelles spatiales, est encore plus délicat à dresser (Stanley et Doyle, 2003; Jørgensen et Renöfält, 2012). Il ne s'agit pas ici de remettre en cause le bien-fondé de la politique de restauration de la continuité, ni de conclure à l'impossibilité d'une prise de décision devant la multiplicité des conséquences, mais bien de souligner la nécessité d'une approche des actions de restauration qui, sans renier ses ambitions, serait probablement plus intégrée, plus en lien avec la diversité des situations et, dans tous les cas, plus explicite dans ces objectifs et ses conséquences.

\section{Conclusion}

La mesure rétrospective des cernes de croissance des arbres de la ripisylve sur deux sites ayant fait l'objet d'une suppression d'obstacle à l'écoulement en 1997 en Basse-Normandie met en évidence une modification significative de la croissance ligneuse suite à la suppression des ouvrages. Dans la majorité des cas, cette modification est une baisse de la croissance. Cependant, cette baisse reste modérée dans la mesure où nous n'observons pas une chute importante de celle-ci comme certains auteurs ont pu l'observer dans d'autres contextes (climat plus sec, abattement plus important de la nappe phréatique). S'il est difficile d'observer une variabilité spatiale sensible dans la réponse des individus, en revanche, toutes les espèces ne répondent pas de la même façon. En effet, sur un des deux sites, la suppression du barrage affecte négativement la croissance des aulnes mais positivement celle des frênes et des tilleuls. Ce résultat est cohérent avec les exigences stationnelles de ces espèces et semble indiquer une baisse du caractère hygrophile des ripisylves. Il souligne également la complexité de l'évaluation globale de la politique de restauration de la continuité écologique sur les cours d'eau. Ecosystèmes aquatiques, écosystèmes riverains, bâti historique, pratiques sociales... autant d'éléments à intégrer par des processus qui réclament conjointement des connaissances scientifiques multi-thématiques et des dispositifs politiques efficients.

\section{Remerciements}

Vincent Bernard et Elise Werthe du Centre de Recherche en Archéologie, Archéosciences, Histoire (UMR6566) pour l'accueil et l'aide à la réalisation des mesures dendrologiques. Les commentaires des relecteurs ont significativement amélioré la qualité du texte et Vincent Dubrenil et Olivier Cantat pour la fourniture de données météorologiques. 


\section{Bibliographie}

Amlin N.M., Rood S.B., 2003. Drought stress and recovery of riparian cottonwoods due to water table alteration along Willow Creek, Alberta, Trees, n 17, p. 351-358.

Astrade L., 1996. La Saône en crue : dynamique d'un hydrosystème anthropisé. Thèse de doctorat en Géographie, Paris IV Sorbonne, $358 \mathrm{p}$.

Astrade L., Bégin Y., 1997. Tree-ring response Populus tremula L. and Quercus robur L. to recent spring floods of the Saône River, France, Ecoscience, n 4, p. 232-239.

Astrade L., Bégin Y., 2003. La dendrochronologie : un outil de diagnostic et d'évaluation du fonctionnement des hydrosystèmes, in Piégay H., Pautou G., Ruffinoni C. (dir.), Les forêts riveraines des cours d'eau, IDF, p. 204-216.

Astrade L., Dufour S., 2010. Dendrochronologie en ripisylve des cernes aux changements hydromorphologiques dans les systèmes fluviaux, Collection EDYTEM, n 11, p. 129-138.

Auble G.T., Shafroth P.B., Scott. M.L., Roelle. J.E., 2007. Early vegetation development on an exposed reservoir : Implication for dam removal, Environmental Management, n³9, p. 806-818.

Beguin Y., Langlais D., Cournoyer L., 1991. Tree ring dating of shore erosion events (Upper st. Lawrence estuary, eastern Canada), Geografiska Annaler, Series A, Physical geography, $\mathrm{n}^{\circ} 73$, p. 53-59.

Bellec P., Lefebvre E., 2012. Plan d'action pour la restauration de la continuité écologique des cours d'eau. Conseil générale de l'Environnement et du Développement durable, rapport n008036 - 01, 86 p. (non publié).

Besnard G., 1987. Écophysiologie de trois essences alluviales et contribution de la nappe phréatique à leur alimentation en eau dans les forêts riveraines du Haut-Rhône français. Thèse de doctorat, Université Joseph-Fourier, Grenoble, 187 p.

Braam R.R., Weiss E.E.J., Burrough P.A., 1987. Spatial and temporal analysis of mass movement using dendrochronology, CATENA, $\mathrm{n}^{\circ} 14$, p. 573-584.

Bravard J.-P., Fagot P., Gadiolet P., Magne M., 1989. Étude de dendrochronologie dans le lit majeur de l'Ain : la forêt alluviale comme descripteur d'une " métamorphose fluviale », Revue de géographie de Lyon, n 64, p. 213-223.

Chiu M.C, Yен C.H., Sun Y.H., Kuo M.H., 2013. Short-term effects of dam removal on macroinvertebrates in a Taiwan stream, Aquatic Ecology, n 47, p. 245-252.

Depoilly D., 2014. Impact de la restauration des continuités écologiques des cours d'eau sur la végétation riveraine. Mémoire de master 2, Université Rennes 2, 36 p.

Disalvo A.C., Hart S.C., 2002. Climatic and stream-flow controls on tree growth in a Western Montane riparian forest, Environmental Management, ${ }^{\circ}$ 30, p. 678-691.

Dubreuil V., Planchon O., Lamy C., Bonnardot V., QuéNoL H., 2012. Le changement climatique dans la France de l'Ouest : observations et tendances, in Dubreuil V., Mérot P., Delahaye D. (dir.), Changement climatique dans l'Ouest, Rennes, PUR, p. 19-30.
Dufour S., Piégay H., 2008. Geomorphological controls of Fraxinus excelsior growth and regeneration in floodplain forests, Ecology, n 89, p. 205-215.

Dufour S., 2005. Contrôles naturels et anthropiques de la structure et de la dynamique des forêts riveraines des cours d'eau du bassin rhodanien (Ain, Arve, Drôme et Rhône). Thèse de doctorat de Géographie, Université de Lyon, 244 p.

Galuszka D.M., Kolb T.E., 2002. Tree growth and regeneration response to climate and stream flow in a species-rich southwestern riparian forest, Western North American Naturalist, $\mathrm{n}^{\circ}$ 62, p. 266-279.

Germaine M.A., Barraud R., 2013. Les rivières de l'Ouest de la France sont-elles seulement des infrastructures naturelles? Les modèles de gestion à l'épreuve de la DCE, Natures Sciences Sociétés, n² 21, p. 373-384.

Golet F.C., Lowny D.J., 1987. Water regimes and tree growth in Rhode Island Atlantic white cedar swamps, in LADERMAN A.D., Atlantic white cedar wetlands, Westview Press, Boulder, CO, USA, p. 91-110.

Horreo J.L., De La Hoz J., Machado-Schiaffino G., Pola I.G., Garcia-VAzQuEz E., 2012. Restoration and enhancement of Atlantic salmon populations: what we have learned from North Iberian rivers, Knowledge and Management of Aquatic Ecosystems, n 402, p. 23.

Hupp C., Dufour S., Bornette G., 2016. Vegetation as a tool in the interpretation of fluvial geomorphic processes and landforms. in Kondolf G.M., Piégay H. (dir.), Tools in fluvial geomorphology. Second edition, Wiley, p. 201-235.

IsAKSSON M., 2010. Response of riparian vegetation to removal of the Kuba dam in Nätraån, Mémoire de Master 2, Umea university, $26 \mathrm{p}$.

Jørgensen D., Renöfält B.M., 2012. Damned if you do, dammed if you don't: debates on dam removal in the Swedish media, Ecology and Society, n ${ }^{\circ}$ 18, p. 18.

Keeland B.D., Conner W.H., Sharitz R.R., 1997. A comparaison of wetland tree growth response to hydrologic regime in Louisiana and South Carolina, Forest Ecology Management, $\mathrm{n}^{\circ}$ 90, p. 237-250.

Kim S.N., Toda Y., Tsujimoto T., 2014. Effects of a Low-Head Dam Removal on River Morphology and Riparian Vegetation: A Case Study of Gongreung River, Journal of Water Resource and Protection, $\mathrm{n}^{\circ}$ 6, p. 1682-1690.

Lubes H., Masson J.-M., Servat E., Paturel J.-E., Kouame B., Boyer J.F., 1994. Caractérisation de fluctuations dans une série chronologique par application de tests statistiques, Étude bibliographique, Programme ICCARE, Rapport n³, ORSTOM, Montpellier, France. Disponible (5 mai 2015) sur [http://www.hydrosciences.fr] (non publié).

MäKinen H., Vanninen P., 1999. Effect of sample selection on the environmental signal derived from tree-ring series. Forest Ecology and Management, $\mathrm{n}^{\circ} 113$, p. 83-89.

Miller P., Vizcaíno P., 2004. Channel response to Dam Removal, Clear Creek, California. Water Resources Center Archives, University of California Water Resources Center, UC Berkeley. Disponible ( $1^{\mathrm{er}}$ mai 2015) sur [http://escholarship.org/uc/item/02v4m1hw] (non publié). 
Mitsch W.J., Rust W.G., 1984. Tree growth responses to flooding in a bottomland forest in northeastern Illinois, Forest Science, $n^{\circ} 30$, p. 499-510.

Moulin B., 2005. Variabilité spatiale et temporelle du bois mort dans le réseau hydrographique de l'Isère à l'amont de Grenoble. Thèse de Doctorat, Université Jean-Monnet SaintÉtienne, 444p.

Munaut A.-V., 1979. La dendrochronologie. Bulletin de l'Association française pour l'étude du quaternaire, $\mathrm{n}^{\circ}$ 16, p. 65-74.

Orr C.H., Stanley E.H., 2006. Vegation development and restoration potential of drained reservoirs following dam removal in Wisconsin, River Research and Applications, $\mathrm{n}^{\circ} 22$, p. 281-295.

Piégay H., Peiry J.-L., Gazelle F., 2003. Effets des ripisylves sur la dynamique du lit fluvial et de son aquifère. in PIÉGAY H., Pautou G., Ruffinoni C. (éd), Les forêts riveraines des cours d'eau : écologie, fonctions et gestion, Institut pour le développement forestier, p. 94-122.

Pizzuto J.E., 2002. The effects of dam removal on river form and process, Bioscience, $\mathrm{n}^{\circ}$ 52, p. 683-691.

Reilly P.W., Johnson W.C., 1982. The effects of altered hydrologic regime on tree growth along Missouri River in North Dakota, Canadian Journal of Botany, n 60, p. 2410-2423.

Rubino D.L., McCarthy B., 2004. Comparative analysis of dendroecological methods used to assess disturbance events, Dendrochronologia, n 21, p. 97-115.

Shafroth P.B., Stromberg J.C., Patten D.T., 2000. Woody riparian vegetation response to different alluvial water table regimes, Western North American Naturalist, $\mathrm{n}^{\circ}$ 60, p. 66-76.

Shaforth P.B., Friedman J.M., Auble G.T., Scott M.L., Braatne J.H., 2002. Potential responses of riparian vegetation removal, BioScience, n ${ }^{\circ}$ 52, p. 703-712.

Singer M.B., Johnstone L., Dufour S., Stella J.C., Piégay H., Wilson R.J.S., 2012. Contrasting water uptake and growth responses to drought in co-occurring riparian tree species, Ecohydrology, n 6, p. 402-412.
Smelko S., Scheer L., 2000. Dendrochronological analysis of diameter growth and increment of pedunculate oak (Quercus robur L.) in Danube floodplain forests, Ekologia (Bratislava), n 19, p. 125-140.

Stanley E.H., Doyle M.W., 2003. Trading off: the ecological effects of dam removal, Frontiers in Ecology and Environment, $\mathrm{n}^{\circ} 1$, p. 15-22.

Stella J.C., Riddle J., Piégay H., Gagnage M., Trémélo M.L., 2013. Climate and local geomorphic interactions drive patterns of riparian forest decline along a Mediterranean Basin river, Geomorphology, n 202, p. 101-114. DOI: 10.1016/j.geomorph.2013.01.013.

Stoffel M., Astrade L., Corona C., Lopez-Saez J., 2012. L'utilisation des cernes de croissance des arbres pour l'étude des événements et des changements morphologiques : intérêts, méthodes et apports des recherches alpines à la dendro-géomorphologie, Géomorphologie : relief, processus, environnement, $\mathrm{n}^{\circ}$ 3, p. 39-60.

Stromberg J.C., Patten D.T., 1996. Instream flow and cottonwood growth in the eastern Sierra Nevada of California, USA, Regulated River: Research and Management, $\mathrm{n}^{\circ}$ 12, p $1-12$.

Tardif J., Bergeron Y., 1997. Comparative dendroclimatological analysis of black ash and two white cedar populations from contrasting sites in the Lake Duparquet region, northwestern Quebec, Canadian Journal of Forest Research, $\mathrm{n}^{\circ} 27$, p. 108-116.

Willms J., Rood B., Willms W., Tyree M., 1998. Branch growth of riparian cottonwoods: a hydrologically sensitive dendrochronological tool, Trees, ${ }^{\circ}$ 12, p. 215-223.

Zitek A., Schmutz S., Jungwirth M., 2008. Assessing the efficiency of connectivity measures with regard to the EUWater Framework Directive in a Danube-tributary system, Hydrobiologia, n 609, p. 139-161. 